\title{
CARTOGRAFIA PRELIMINAR DOS MUSEUS DA DIASPORA AFRICANA NO MUNDO 2018
}

\section{Rafael Sanzio Araújo dos Anjos Richard Denis}

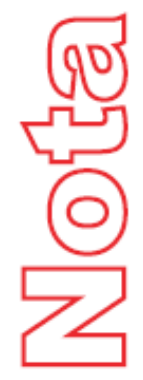

p. $01-20$

\section{Revista}

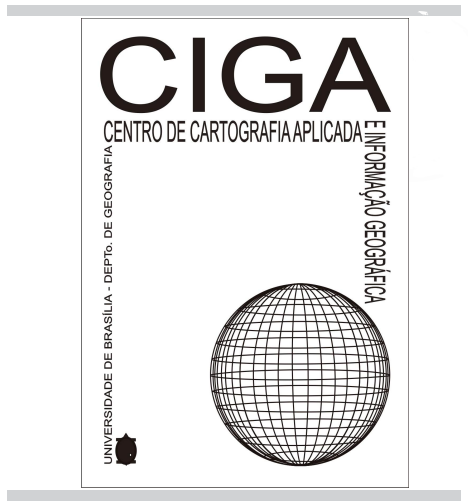

Revista Eletrônica:

Tempo - Técnica - Território, V.9, N.3 (2018), 01:20

ISSN: $2177-4366$
Como citar este ar tigo:

Sanzio, R. Richard Denis. CARTOGRAFIA PRELIMINAR DOS MUSEUS DA

DIASPORA AFRICANA NO MUNDO 2018. Revista Eletrônica: Tempo - Técnica

- Território, v.9, n.3 (2018), p. 1:20

ISSN: 2177-4366.

Disponível em:

http://periodicos.unb.br/index.php/ciga/issue/view/1916

Este obra está licenciado com uma Licença Creative Commons Atribuição-NãoComercial 4.0 Internacional. 


\section{CARTOGRAFIA PRELIMINAR DOS MUSEUS DA DIASPORA AFRICANA NO MUNDO 2018}

\section{NOTA TÉCNICA}

Rafael Sanzio Araújo dos Anjos (*), Richard Denis, Izabella C. de A. dos Anjos (**) Email:cartografia@unb.br Site:www.unb.br

(*) Geógrafo, Doutor em Informações Espaciais Pós-Doutorado Cartografia Étnica. Prof. Titular da Universidade de Brasília / Diretor do Centro de Cartografia Aplicada e Informação Geográfica do Depto. de Geografia - UnB

(**) Pesquisadora Colaboradora do CIGA e Estagiários membros do Centro de pesquisa na UnB

RESUMO: A Cartografia da Geopolítica dos Museus da Diáspora Africana no Mundo dedica-se a representar cartograficamente a distribuição espacial dos museus com acervos da diáspora africana ao redor do mundo. Dedica-se a dar visibilidade aos museus da diáspora e concentrar informações geográficas acerca dos mesmos. Tem o proposito de, além de expor a geopolítica dos museus, apresentar um fichamento técnico-informativo acerca dos museus, seus acervos, atividades ofertadas e localização.

ABSTRACT:The Cartography of the Geopolitics of the Museums of the African Diaspora in the World is dedicated to cartographically representing the spatial distribution of museums with collections from the African diaspora around the world. It is dedicated to giving visibility to the museums of the diaspora and concentrating geographic information about them. It has the purpose of, besides exposing the geopolitics of the museums, present a technical-informative file about the museums, their collections, offered activities and location.

\section{1 - A CARTOGRAFIA, O ORDENAMENTO DO TERRITÓRIO.}

A informação espacial referente à forma de ocupação que se processa no território constituiu uma das mais estratégicas para responder e informar sobre as inúmeras indagações do que está acontecendo, do que pode acontecer e do que aconteceu no espaço geográfico. Nesse sentido, o mapeamento das ocupações e dos espaços como ferramenta de armazenamento e comunicação de dados geográficos têm experimentado significativos avanços nas fontes geradoras das informações, principalmente nas últimas décadas, com os produtos de sensoriamento remoto de última geração e as tecnologias computacionais para manipulação e referencia mento da informação territorial. Como ponto inicial do desenvolvimento da pesquisa, abordamos brevemente os pressupostos instrumentais e metodológicos utilizados na confecção da cartografia temática da Geopolítica da Diáspora Africana no Mundo. Com este trabalho buscamos contribuir efetivamente para a ampliação do conhecimento sobre os acervos da diáspora africana espalhados por todo o globo, evidenciando a geopolítica dos museus africanos e sua distribuição espacial no âmbito internacional. 


\section{2 - O CIGA.}

Não podemos perder de vista que a Universidade de Brasília (UnB) consolidou-se como uma das melhores instituições de ensino, pesquisa e extensão do Brasil, reconhecida pela qualidade na sua produção acadêmica e pela elevada qualificação de seu corpo docente. Seguindo as suas referências básicas, o Departamento de Geografia e o Programa de Pós Graduação em Geografia da UnB constituíram, em 1999, o Centro de Cartografia Aplicada e Informação Geográfica (CIGA), com o propósito de dar maior amplitude nos projetos e parcerias em andamento, a partir da integração da educação, pesquisa e atendimento às demandas da sociedade civil. O CIGA tem uma estrutura física organizada com equipamentos básicos para o desenvolvimento de atividades direcionadas para o manuseio de ferramentas geográficas voltadas para a educação geográfica e o planejamento-gestão do território. É importante destacar que os sistemas de informação territorial tem presenciado uma forte difusão de dados e tornado acessíveis outras possibilidades de representação do espaço geográfico. Por outro lado, as demandas para compreensão e resolução das complexas questões da dinâmica da sociedade são crescentes e a cartografia se confirma como uma das disciplinas melhor colocadas para responder e informar sobre o que aconteceu, o que está acontecendo de fato e o que pode acontecer no território, ou seja, as representações cartográficas possibilitam trabalhar com o passado, o presente e o futuro próximo do território. Após estes ano de atividades, o CIGA consolida a sua vocação de referência local, regional e nacional na produção técnica de aplicabilidades e alcance social das ferramentas geográficas ligadas à cartografia, sensoriamento remoto e sistemas de informação geográfica.

\section{3 - O PROJETO GEOAFRO}

O Projeto Geoafro dedica-se a retratar a geopolítica dos museus da diáspora africana no espaço mundial. Condensando dados espaciais e informacionais acerca dos referidos acervos. A ideia o projeto é dar visibilidade a distribuição espacial dos museus expositores da cultura material africana vinculada à diáspora. $\mathrm{O}$ projeto se dedica a coletar e processar informações geográficas acerca de tais acervos com o objetivo de simplificar o acesso a informação espacial referente a localização e acesso aos mesmos. Ademais, o projeto se dedica também a levantar informações especificas acerca da historia dos museus pesquisados e do acervo diasporico neles exposto.

\section{4 - A REPRESENTAÇÃO CARTOGRÁFICA DA GEOPOLÍTICA DOS ACERVOS DA DIÁSPORA.}

O presente projeto se destina a representar cartograficamente a distribuição espacial dos museus da Diáspora Africana no mundo, evidenciando a geopolítica existem por detrás da exposição e visibilidade dos acervos referentes ao processo diasporico. Na presente nota serram apresentados os processos desenvolvidos na elaboração do mapa da 
distribuição espacial dos acervos ao redor do mundo e na confecção das fichas informativas referentes a cada museu. Em um primeiro momento será explicado o processo de atualização pelo qual as fichas passaram entre janeiro e fevereiro de 2017, em seguida apresentamos as mudanças metodológicas que tivemos que adotar para sanar alguns problemas técnicos referentes à numeração dos museus. Mais a frente esta um modelo de estrutura padrão das fichas, que serve para exemplificar como as informações estarão distribuídas em todas as fichas. Em seguida expomos o procedimento empregado para referenciar espacialmente os museus nas fichas, com suas respectivas coordenadas e imagens aéreas. Por fim, apresentaremos o mapa final que conglomera a localização de todos os acervos fichados. A figura 1, fragmento gráfico do mapa final, represente o quantitativo da distribuição dos museus da diáspora por continente.

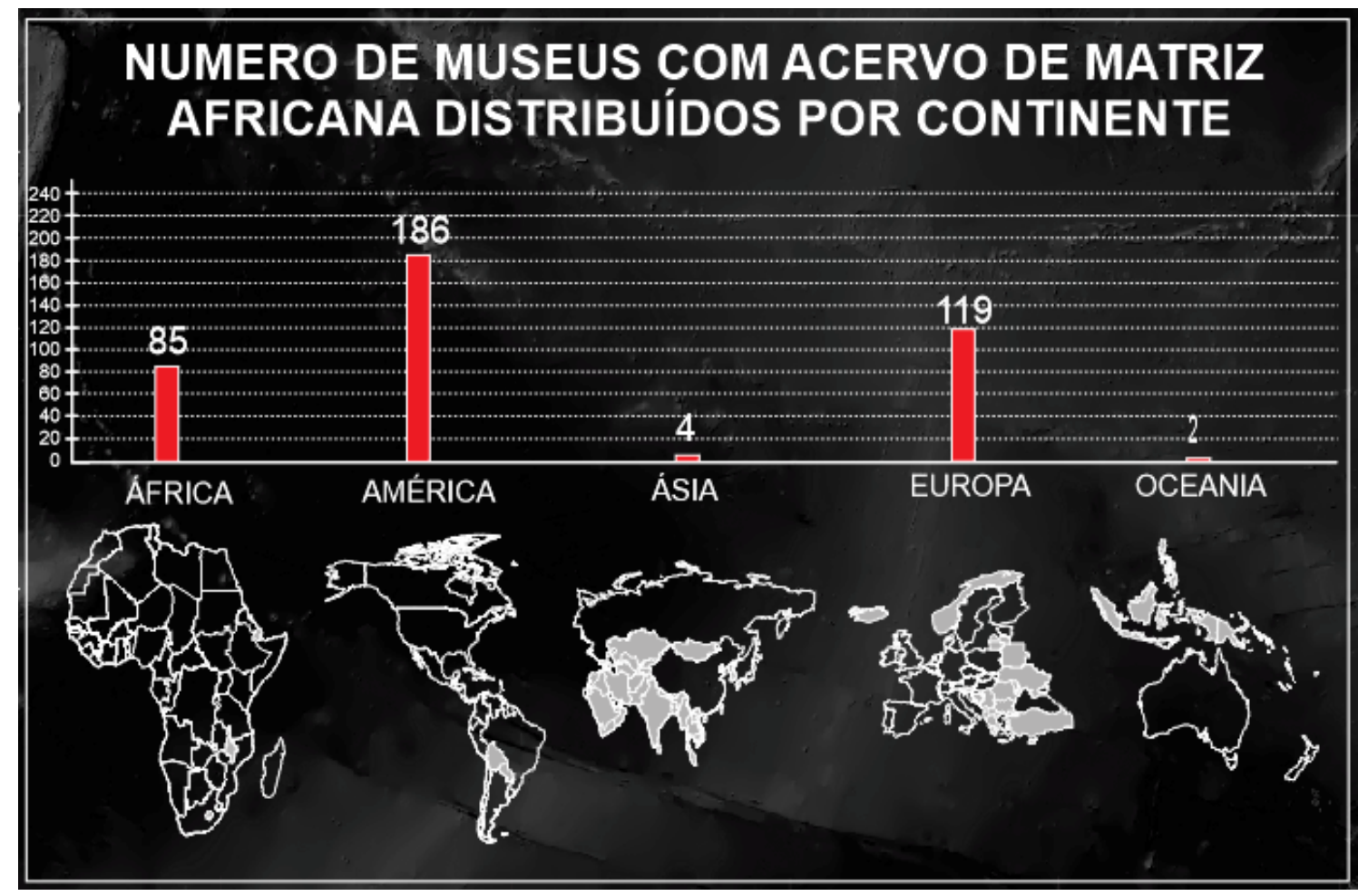

(Imagem 1)

\section{5 - ATUALIZAÇÃO DAS FICHAS}

O trabalho começou tendo por base o mapa da Geopolítica dos Museus com acervo Africano no Mundo e os dados utilizados para sua confecção. Tal base de dados serviu como um ponto de partida para a aglomeração de mais dados acerca de acervos e a base cartográfica serviu de inspiração para o mapa final dos museus da diáspora. Devido à mudança metodológica foi necessário renumerar todas as fichas segundo o novo padrão de numeração. Feito isso, adicionou-se também a seguinte nota de roda pé nas fichas: “Este número é referente à numeração da sistematização dos registros dos museus com acervo de matriz africana por continentes no planeta. Esta numeração faz parte, também, do mapa temático Geopolítica dos Museus com Acervo Africano no Mundo, elaborado no bojo do mesmo Projeto GEOAFRO. Brasília - Brasil. 2017 E-mail: 
quilombo@unb.br'’. Além das atualizações referentes à mudança metodológica, atualizou-se também o mapa base usado para localizar os museus no mundo. O mapa base se mostrava obsoleto, pois ainda não possui as fronteiras do recém-independente Sudão do Sul, oque tornava as fichas de certa forma desatualizadas. Para sanar esse problema todas as fichas passaram por um processo de atualização que consistiu em adicionar ao mapa de localização mundial a fronteira sul do Sudão, que divide o território do Sudão do território do Sudão do Sul.

\section{ESTRUTURAÇÃO DAS FICHAS}

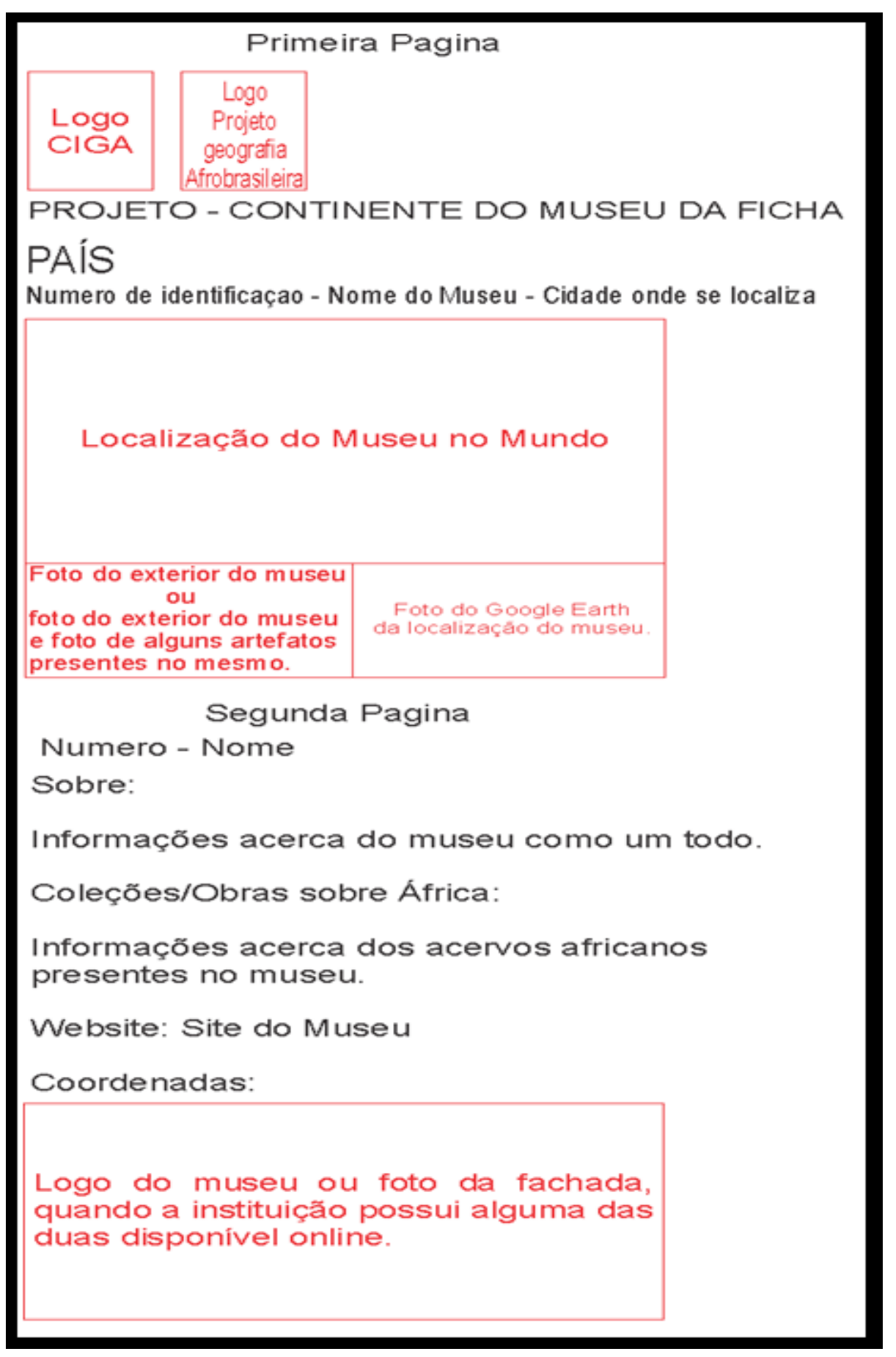

(Modelo de ficha esquematizada) 


\section{7 - REFERENCIAMENTO ESPACIAL DOS MUSEUS}

As fichas dos museus estão passando por um processo de atualização de informações e georreferenciamento espacial. Os museus estão sendo localizados através do Google Maps e do Google Earth e os dados de suas posições geográficas estão sendo adicionadas as fichas.

Em um primeiro momento os museus estão sendo localizados no Google Maps, uma foto aérea ou mapa virtual do museu e de suas proximidades foi adicionada as fichas que não a possuem. Nas fotos aéreas estão sendo ''hiperlinkados' os endereções eletrônicos referentes a localização do museu no Google Maps. Além disso, ao final das fichas estão sendo adicionadas as Coordenadas dos museus.

Esse procedimento é a primeira fase da obtenção de dados geográficos dos museus e integração dessas informações online com as fichas. Segue abaixo ilustração de todas as etapas.

7.1 -A obtenção da localidade no Google Maps pode ser vista na imagem 2.

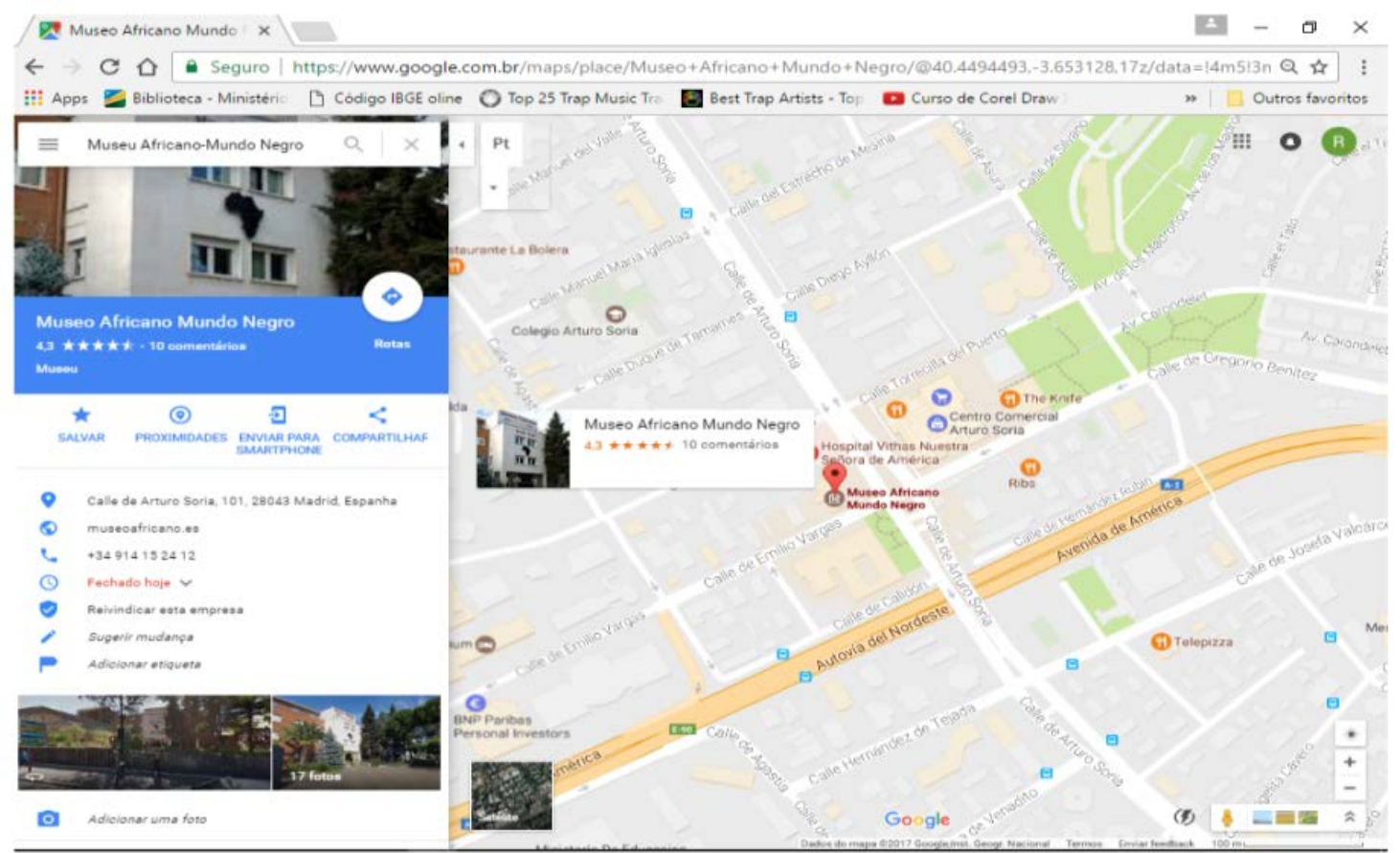

(Imagem 2) 
7.2 - Adição de hiperlink de comunicação entre a ficha e a localização do museu, pode ser vista nas imagens 3 e 4 .

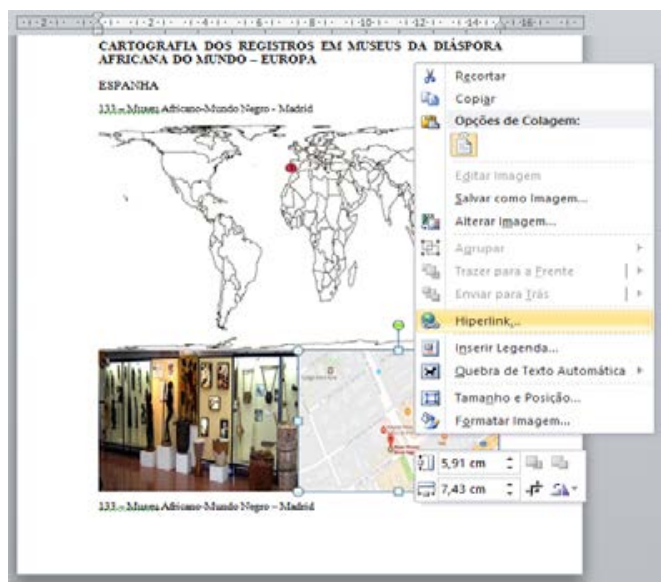

(Imagem 3)

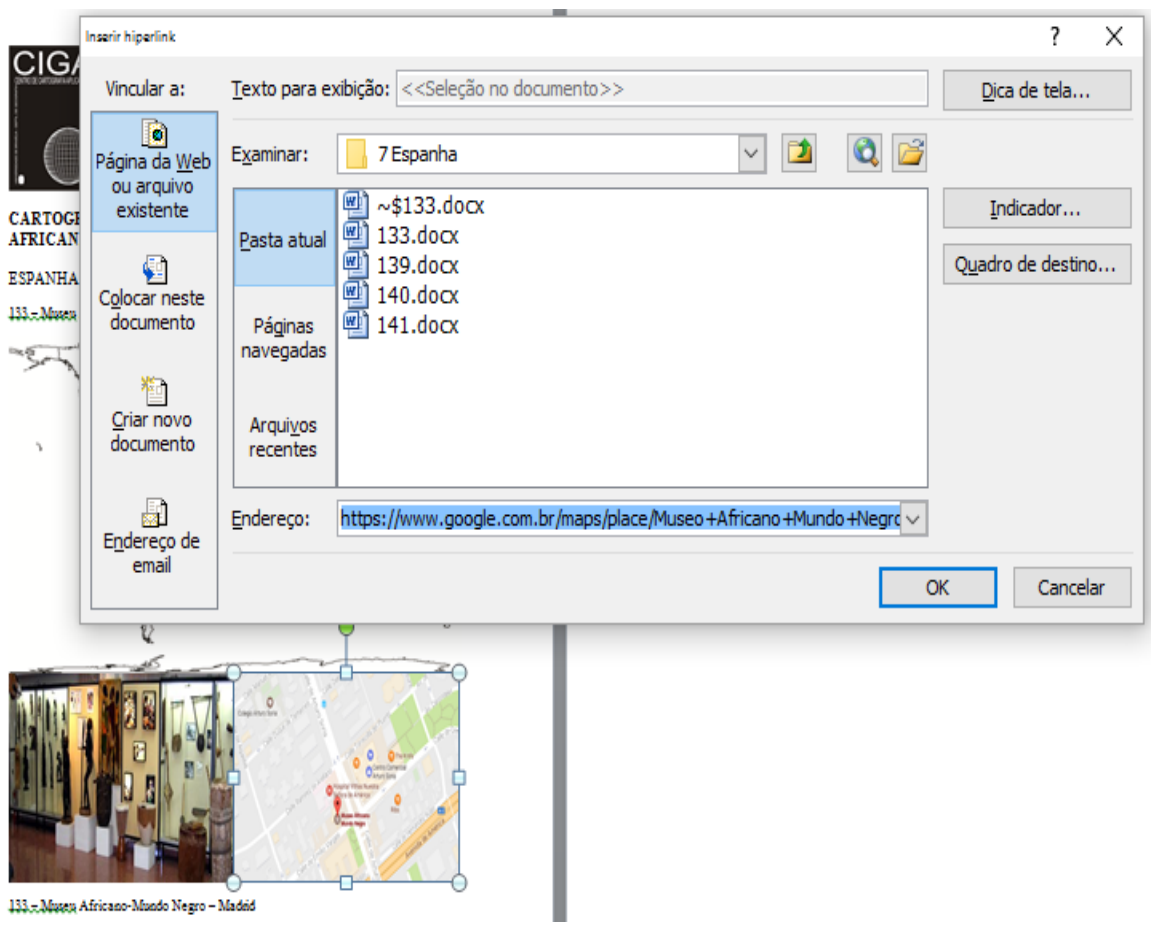

(Imagem 4) 
7.3 - Adição das coordenada obtidas por meio do Google Earth. Tal processo pode ser visto nas imagens 5 e 6.

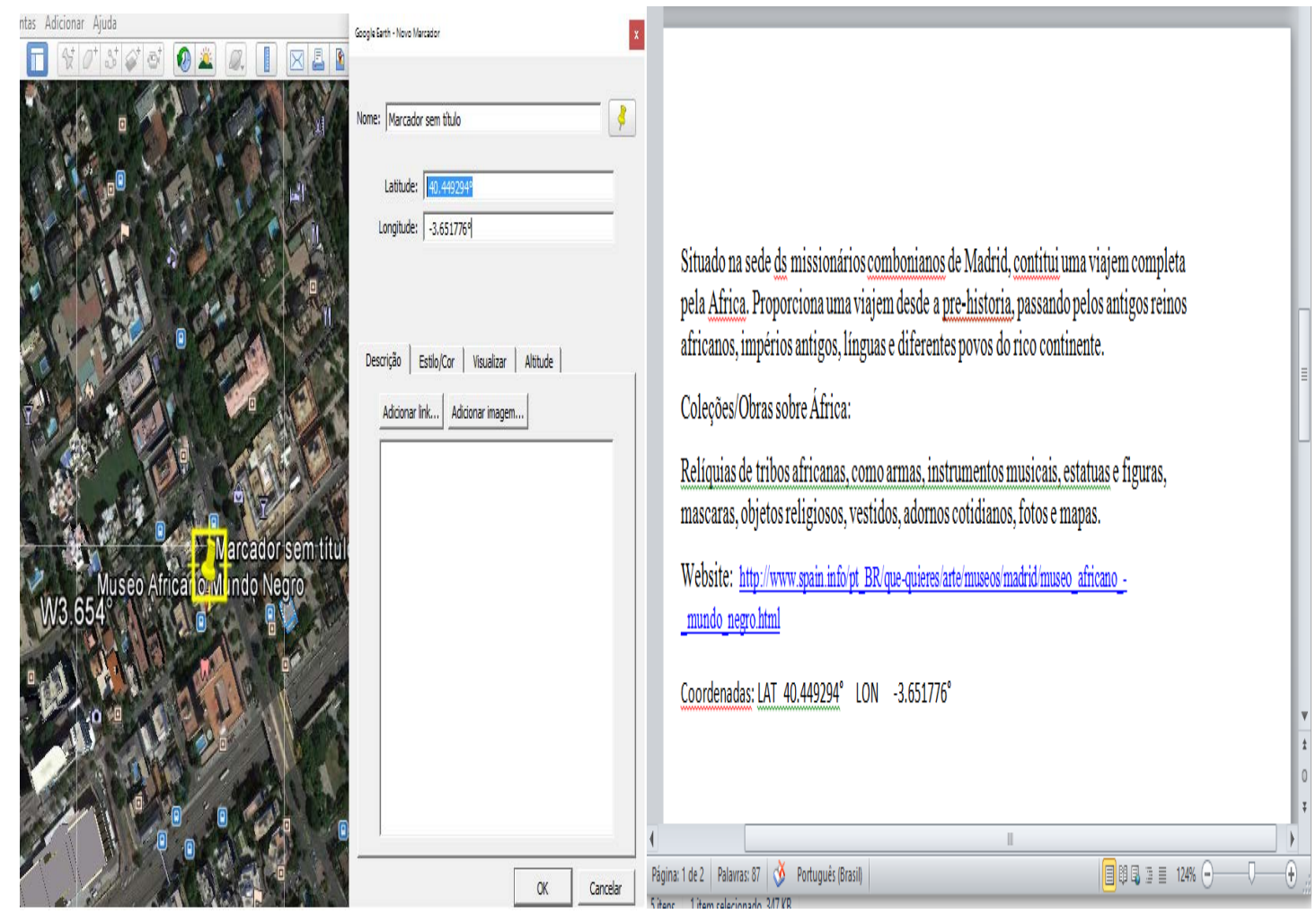

(Imagem 5 e 6)

\section{8 - ELABORAÇÃO DO PRODUTO CARTOGRÁFICO}

O produto final do projeto trata-se de um mapa geral que reúne todos os museus pesquisados em uma única carta. O mapa final foi desenvolvido utilizando-se a Projeção Arno Peters adaptada, a localização dos pontos foi feita de forma aproximada com base nas coordenadas referentes a cada um deles. Com o intuito de fornecer á sequencia numérica um padrão geograficamente racional as numerações do mapa foram todas alteradas. As numerações no mapa representam os museus com acervo do continente africano ordenados numericamente dentro de seus continentes. A numeração começa de forma crescente do norte para o sul, crescendo por países da esquerda para a direita, quando com mais de um país na mesma latitude. Dentro dos países a distribuição dos museus não segue nenhuma sequência geograficamente orientada. Esses números fazem referencia a numeração dos museus no Quadro dos Principais Museus com Acervo da Diáspora Africana por Continente. Devido a tal mudança alguns itens tiveram de ser alterados em todas as fichas. O produto cartográfico final pode ser visto na imagem 7. Na imagem 8 temos um zoom dado no mapa final em uma região de elevada densidade de museus. Na imagem 9 tem-se o verso do mapa final, tal face conta com a lista completa dos museus localizados no mapa, sua numeração, cidade e país onde se localiza, além das fontes onde foram obtidas todas as informações cartograficamente representadas no mapa e qualitativamente transcritas nas fichas informativas. 


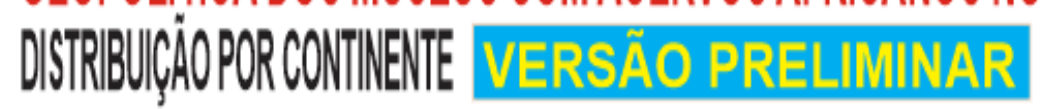
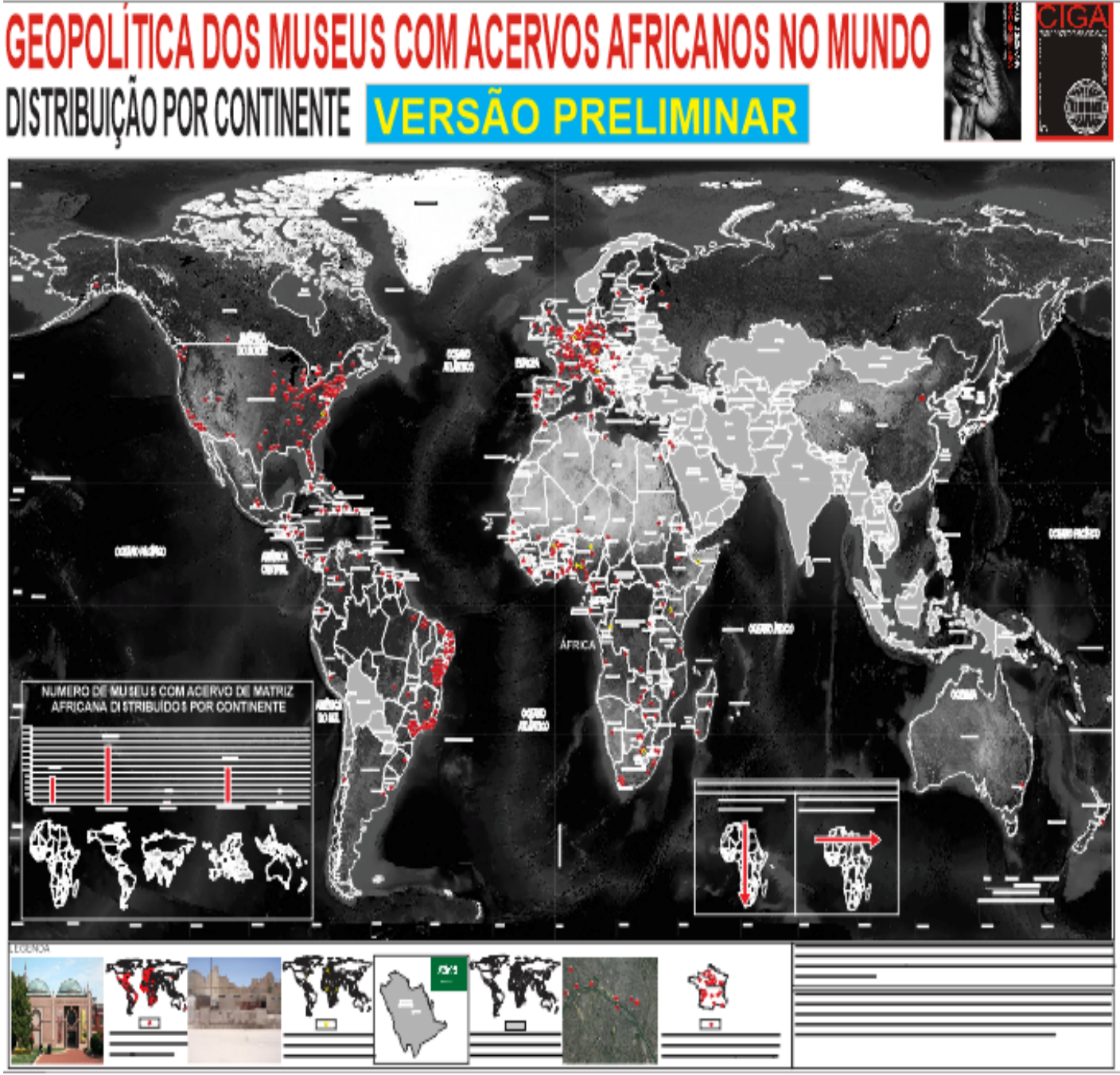

(Imagem 7)

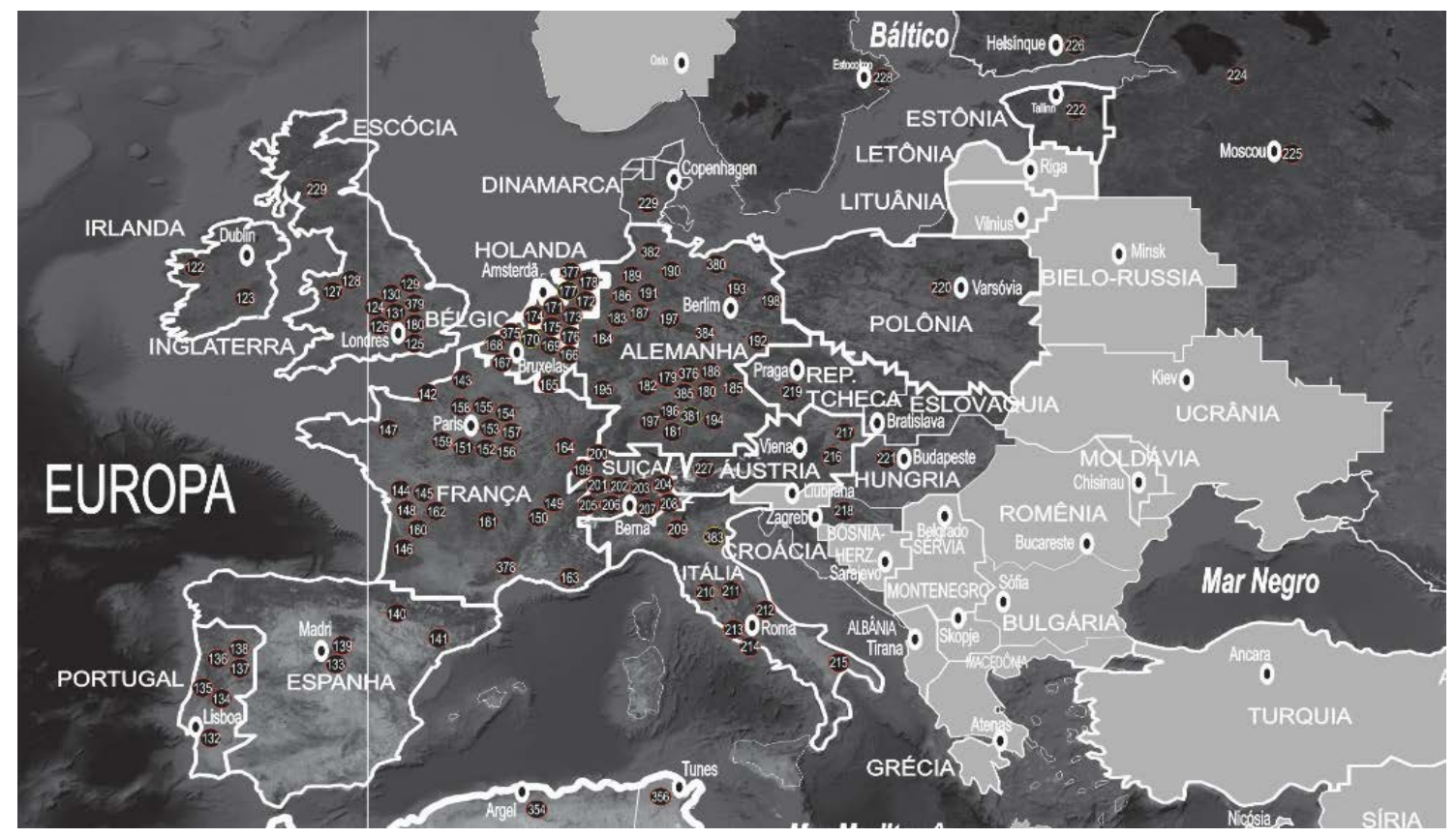

(Imagem 8) 


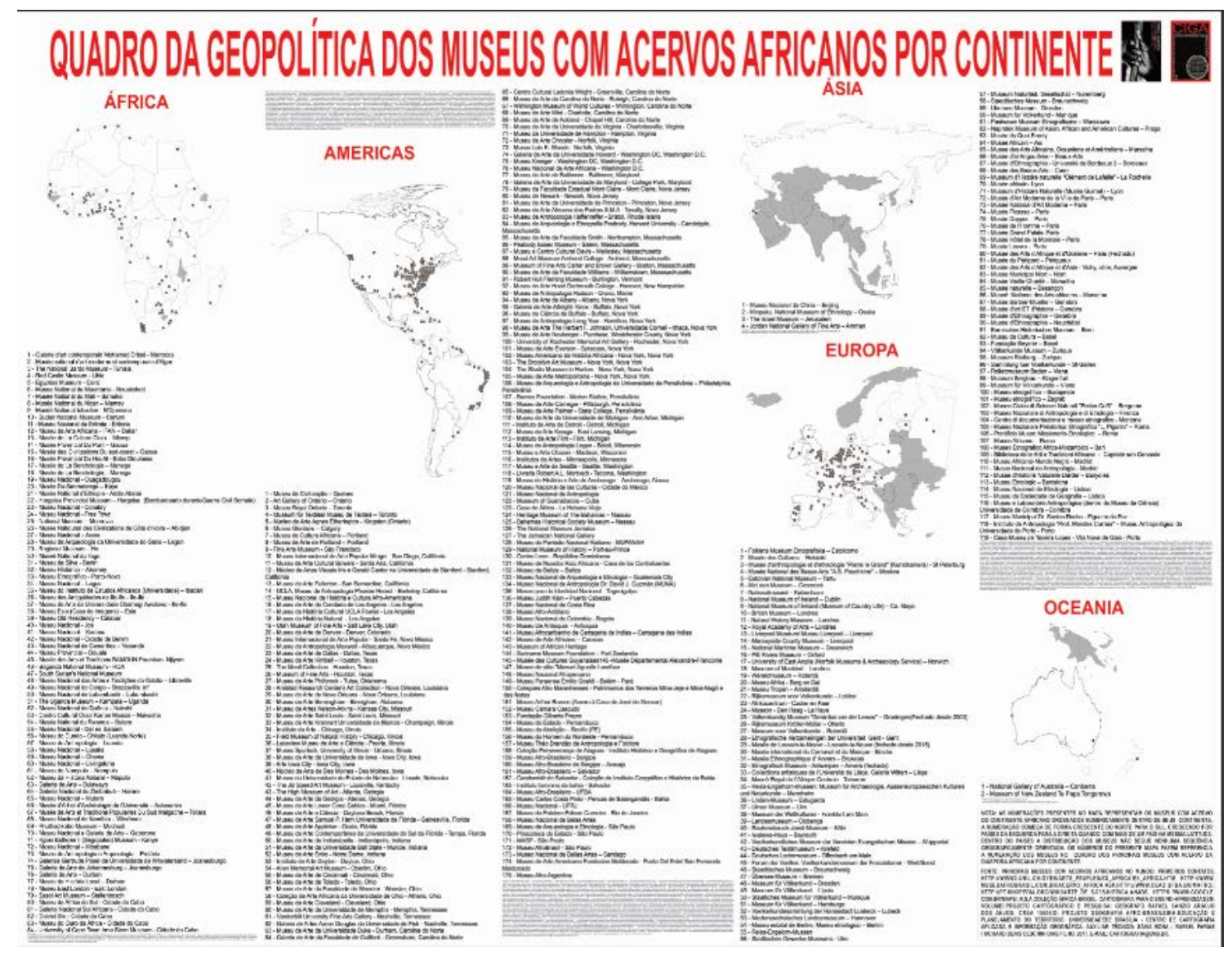

(Imagem 9)

\subsection{Numeração}

A sequencia numérica foi padronizada de forma que cada continente conta com uma sequencia numérica independente e ordenada de forma geograficamente logica. A contagem é feita do norte ao sul de cada continente, sendo que quando os museus de encontram na mesma latitude a progressão numérica ocorre do oeste para o leste. Tal metodologia é didaticamente representada visualmente no mapa final através da imagem 10.

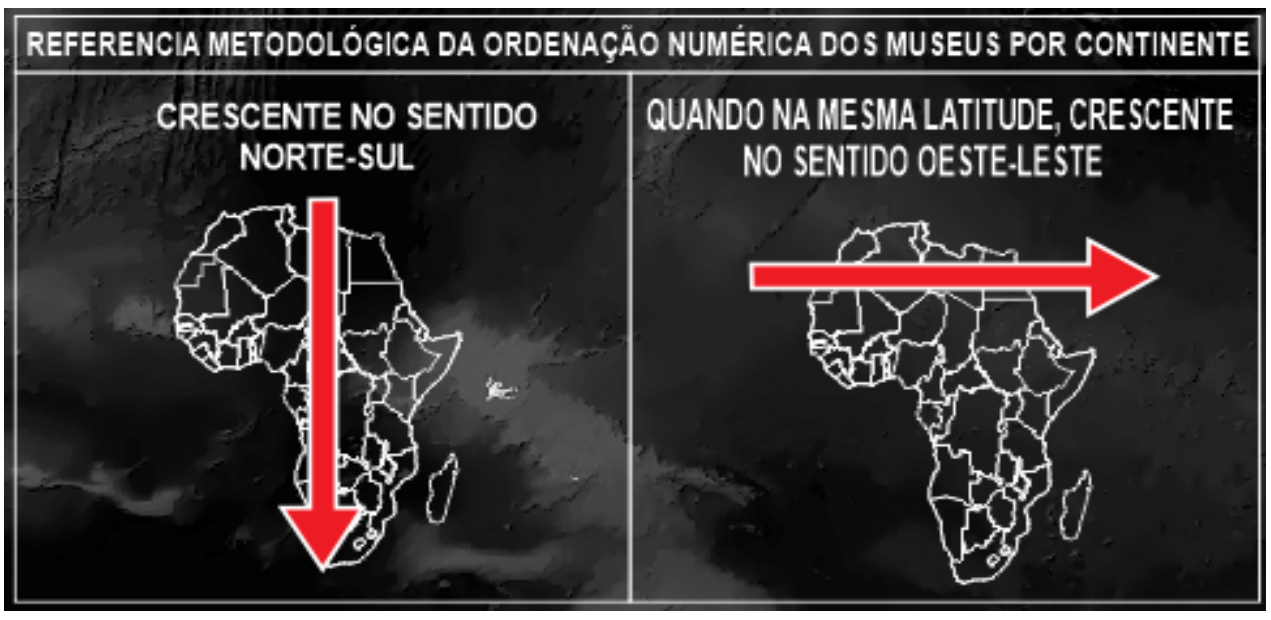

(Imagem 10) 
8.2 Legenda

A legenda busca informar quais paises possuem acervos, quais não possuem, quais museus no mundo estão operacionais e quais aqueles que se encontram por qualquer motivo fechados ao publico. A imagem 11 traz um recorte de todas as informações contidades na legenda do mapa final.

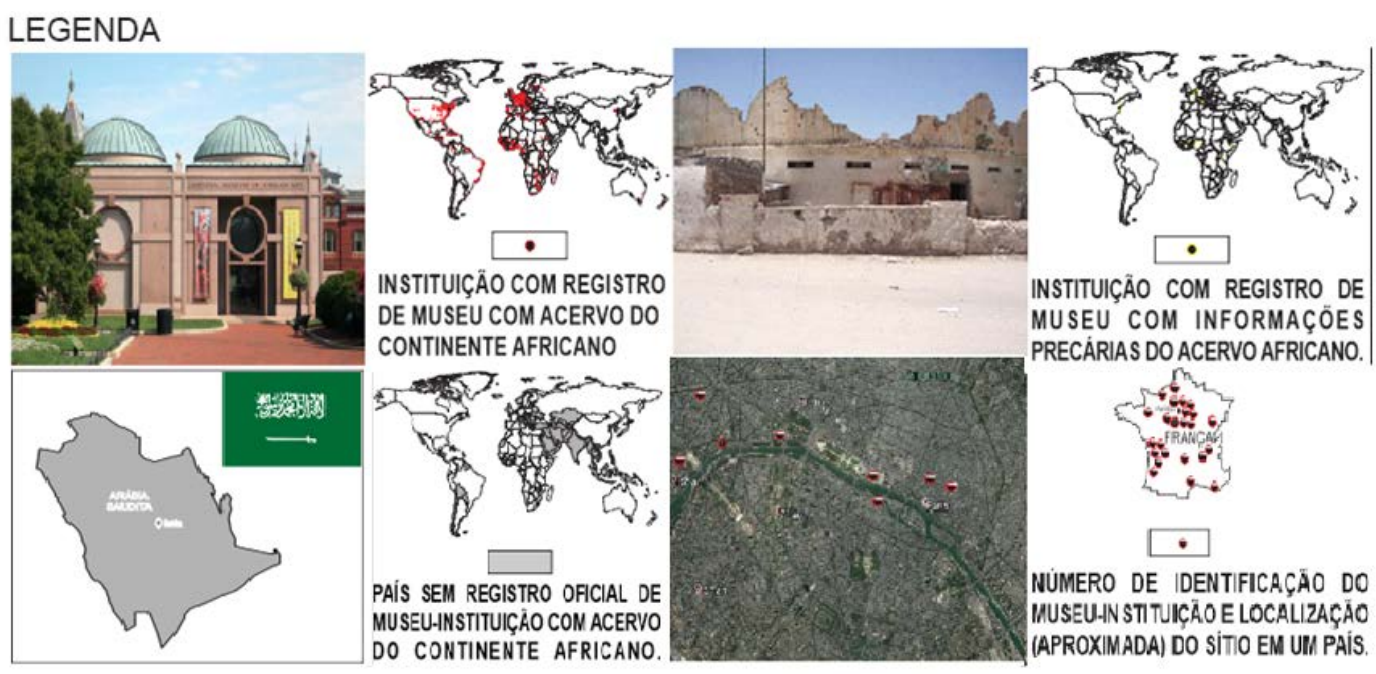

(Imagem 11)

\subsection{Link do Metadado Espacial}

O metadado do mapa final desenvolvido no projeto referido pode ser acessado no link que segue a baixo.

http://164.41.109.9:8080/geonetwork/srv/por/catalog.search\#/metadata/2648d2cc-b8604a60-a62a-c7c6eb27ade3

\section{9 - ARCERVOS VIRTUAIS ACESSADOS PARA DESENVOLVIMENTO DO TRABALHO.}

Todos disponíveis e acessados entre janeiro e abril do ano de 2017.

África

http://www.minculture.gov.ma/fr/index.php?option=com_content\&view=article\&id=184\%3Ag alerie-dart-contemporain-mohamed-drissi-\&catid=43\&Itemid=129\&lang=fr

http://www.mama-dz.com/index.php

http://www.patrimoinedetunisie.com.tn/eng/musees/bardo.php

https://www.temehu.com/Cities_sites/museum-of-tripoli.htm

http://www.petitfute.com/v52566-nouakchott/c1173-visites-points-d-interet/c958

musee/275650-musee-national.html

http://www.museebamako.org/ 
http://www.infos-niger.com/tourisme/niamey/

http://www.petitfute.com/v45655-n-djamena/c1173-visites-points-d-interet/c958musee/416560-musee-national.html\#l7uRdEP0Cq2XEDEz.99

http://www.shabait.com/about-eritrea/art-a-port/6100-the-national-museum-of-eritrea https://www.expedia.com.br/Museu-IFAN-De-Arte-AfricanaDacar.d6118086 .Guia-de-Viagemarticles/entry/article-southafrica.net-gold-of-africa-museum

http://www.irmastern.co.za/

http://artmap.co.za/174/sasol+art+museum+university+of+stellenbosch+art+gallery/

http://www.iziko.org.za/museums/south-african-national-gallery

http://www.districtsix.co.za/

http://www.southafrica.net/za/en/

http://www.ecotour-voyage-nature.com/le-senegal-nature-et-culture/merveilles-naturelles-etculturelles/211-le-hutendunkaye-ou-le-musee-de-la-culture-traditionnelle-diola-de-mlomp-lesfacettesde-la-culture-de-kassa

http://www.africultures.com/php/index.php?nav=structure\&no=6374

http://lefaso.net/spip.php?article31993

http://bobo-dioulasso.net/Musee-provincial-du-Houet

http://musicinafrica.net/directory/national-museum-music-ouagadougou-burkina-faso?

language $=$ en

http://www.musee-manega.bf/fr/

http://www.burkinatourism.com/Le-Musee-National-du-Burkina-Faso-Ouagadougou.html http://www.petitfute.com/v55624-kaya/c1173-visites-points-d-interet/c958-musee/326274musee-de-kaya.html\#AVSzPoFPMu2Lc1qq.99

http://www.monnuage.fr/point-d-interet/musee-national-dethiopie--a238781

http://unesdoc.unesco.org/Ulis/cgibin/ulis.pl?catno=71666\&set=49F6C8FF_1_8\&gp=0\&lin=1 http://www.petitfute.com/v53060-conakry/c1173-visites-points-d-interet/c958-musee/352614musee-national.html

http://www.sierraleoneheritage.org/museum/

http://www.lonelyplanet.com/liberia/monrovia/sights/museums-galleries/liberia-nationalmuseum

http://civ.abidjan.net/index.php/visiter/musees/81-musee-des-civilisations-de-cote-d-ivoire http://www.ghanamuseums.org/national-museum.php

http://www.ug.edu.gh/archaeology/about/brief history http://www.ghanamuseums.org/volta-museum.php http://www.visitcapitalcity.com/africa/Porto-Novo-Benin http://webcache.googleusercontent.com/search?q=cache:http://epaprema.net/abomeyGB/\&gws $\underline{\mathrm{rd}=\mathrm{cr} \& \mathrm{ei}=1 \mathrm{kTvVvCPE} 8 \mathrm{yswgSj0ouoDg}}$ http://www.visitcapitalcity.com/africa/Porto-Novo-Benin http://www.dasiko.com/city/lagos/attractions/national-museum-lagos/ http://www.museum.com/jb/museum?id=23891 http://ui.edu.ng/africanstudies http://ics.oauife.edu.ng/museum.php http://www.tripatini.com/profiles/blogs/national-museum-esie-the http://afrotourism.com/attraction/old-residency-museum/ http://global.britannica.com/place/Jos-Museum https://unravellingnigeria.com/tag/kaduna-national-museum/ http://www.nigerianobservernews.com/24012014/features/features7.html\#.Vu9E6 krLIU http://www.unesco.org/webworld/ram/17b.HTM 
http://www.petitfute.com/v46011-douala/c1173-visites-points-d-interet/c958-musee/161298musee-maritime-national.html http://www.petitfute.com/v46032-foumban/c1173-visites-points-d-interet/c958-musee/161163musee-des-arts-et-traditions-bamoun.html http://www.iexplore.com/articles/travel-guides/africa/central-african-republic/attractions http://www.lbcld.org/south-sudans-national-museum/ http://www.gabonart.com/visites-virtuelles/musee-virtuel-des-arts-et-traditions http://www.unesco.org/culture/natlaws/media/pdf/congo/congo_decret6845_freorof.pdf http://www.katanga-tourisme.org/musee-national-de-lubumbashi/ http://afrotourism.com/attraction/uganda-national-museum/ http://www.museums.or.ke/content/blogcategory/11/17/ http://www.olpopongi-maasai.com/ http://museum.gov.rw/index.php?id=27 https://www.expedia.com.br/Museu-Nacional-Da-Tanzania-Dar-Es-Salaam.d6120040.Guia-deViagem http://www.welcometoangola.co.ao/_dundo_museum2\&ctd=352 http://www.museuantropologia.angoladigital.net/ http://www.zambiatourism.com/destinations/historic/museums http://www.zambiatourism.com/destinations/historic/museums http://www.zambiatourism.com/destinations/historic/museums http://viajar.sapo.mz/descubra-o-pais/lazer-e-cultura/museu-nacional-de-etnologia http://www.mmo.co.mz/museu-de-historia-natural-100-anos http://www.nationalgallerybyo.com/ http://www.nationalgallery.co.zw/ http://www.nationalgallery.co.zw/mutare/ http://www.univ-antananarivo.mg/Institut-de-Civilisation-Musee-d http://besthotel-madagascar.com/guide-de-tulear http://www.namibian.org/travel/museums/national_museum_namibia.html http://www.botswanatourism.co.bw/destination/mochudi http://www.touristlink.com.br/botswana/museu-nacional/overview.html http://afrotourism.com/attraction/swaziland-national-museum/ http://www.unisa.ac.za/Default.asp?Cmd=ViewContent\&ContentID=912 https://www.wits.ac.za/wam/newwamsite/learn/our-history/ http://www.gauteng.net/attractions/johannesburg_art_gallery/ http://www.durban.gov.za/city_services/parksrecreation/durban_art_gallery /Pages/default.aspx http://www.durban.gov.za/City_Services/ParksRecreation/museums/Local_History_Museums/P ages/default.aspx http://www.elmuseum.za.org/ http://artmap.co.za/174/sasol+art+museum+university+of+stellenbosch+art+gallery/

\section{America}

https://www.mcq.org/en

http://www.ago.net/home http://www.rom.on.ca/en http://www.textilemuseum.ca/home http://agnes.queensu.ca/ 
http://www.glenbow.org/index.cfm

http://www.museumafricanculture.org/\#!about/aboutPage

http://www.portlandmuseum.org/

https://www.famsf.org

http://www.mingei.org/

http://www.bowers.org/

http://museum.stanford.edu/

http://raffma.csusb.edu/index.html

http://hearstmuseum.berkeley.edu/

http://nmaahc.si.edu/

http://www.lacma.org/

http://www.fowler.ucla.edu/

http://www.nhm.org/site/

http://umfa.utah.edu/

http://denverartmuseum.org/

http://www.internationalfolkart.org/

https://www.unm.edu/ maxwell/

https://www.dma.org

https://www.kimbellart.org/

https://www.menil.org/

http://www.mfah.org/

https://philbrook.org/

http://www.amistadresearchcenter.org/

https://noma.org/

http://www.artsbma.org/

http://www.nelson-atkins.org/

http://www.slam.org/

http://www.kam.illinois.edu/

http://www.artic.edu/

https://www.fieldmuseum.org/

https://www.peoriariverfrontmuseum.org

http://spurlock.illinois.edu/

https://uima.uiowa.edu/

http://www.artsiowacity.org/

http://www.desmoinesartcenter.org/

http://museum.unl.edu/

http://www.speedmuseum.org/

https://www.high.org/

https://georgiamuseum.org/

http://www6.miami.edu/lowe/

http://www.moas.org/main.cfm

http://www.harn.ufl.edu/

http://www.appletonmuseum.org/

http://www.graphicstudio.usf.edu 
http://www.imamuseum.org/

http://www.daytonartinstitute.org/

http://www.oberlin.edu/amam/default.html

http://www.cincinnatiartmuseum.org/

http://www.toledomuseum.org/

https://www.wooster.edu/arts/museum/

https://www.ohio.edu/museum/art/collections/african.cfm

http://www.clevelandart.org/

http://www.memphis.edu/amum/

https://as.vanderbilt.edu/gallery/

http://www.fisk.edu/services-resources/fisk-university-galleries

http://nasher.duke.edu/

http://library.guilford.edu/art-gallery/

http://www.ecu.edu/cs-studentaffairs/lwcc/

http://ncartmuseum.org/

http://library.uncw.edu/museum/

https://www.mintmuseum.org/

http://ackland.org/

http://www.virginia.edu/artmuseum

http://museum.hamptonu.edu/

http://www.chrysler.org/

http://www.galleryofart.howard.edu/

https://www.kreegermuseum.org/

http://africa.si.edu/

https://artbma.org/

http://www.artgallery.umd.edu/

https://www.montclair.edu/arts/art-and-design/academic-programs/graduate-programs /museum-management-ma/

http://www.newarkmuseum.org/-artmuseum.princeton.edu

http://smafathers.org/

https://www.brown.edu/research/facilities/haffenreffer-museum/

https://www.peabody.harvard.edu/collections

http://www.smith.edu/artmuseum/

http://www.pem.org/

https://www.wellesley.edu/davismuseum/

https://www.amherst.edu/museums/mead

http://www.mfa.org/

https://wcma.williams.edu/

http://www.uvm.edu/ fleming

http://hoodmuseum.dartmouth.edu/\#

http://umaine.edu/hudsonmuseum

http://www.albanymuseum.com/

http://www.albrightknox.org/

http://www.sciencebuff.org/site/

https://www.longyear.org/ 
http://museum.cornell.edu/ https://www.neuberger.org/ https://mag.rochester.edu/ http://www.everson.org/ http://www.theaamuseum.org/ https://www.brooklynmuseum.org/ http://www.studiomuseum.org/ http://www.metmuseum.org/ https://www.penn.museum/ http://www.barnesfoundation.org/collections/art-collection/ http://www.cmoa.org/ http://palmermuseum.psu.edu/ http://www.umma.umich.edu/ http://www.dia.org/ http://broadmuseum.msu.edu/ http://www.flintarts.org/ https://www.beloit.edu/logan/ http://www.chazen.wisc.edu/ http://new.artsmia.org/ http://www.seattleartmuseum.org/ https://www.plu.edu/library/ https://www.anchoragemuseum.org/ http://www.museodelasculturas.mx/ http://www.mna.inah.gob.mx/index.html http://www.webhavana.com/en/museum_guanabacoa.html http://www.visitarcuba.org/museo-casa-de-africa http://www.thebahamasweekly.com/publish/arts-and-culture/Heritage_Museum_of_ The_Bahamas_Newest_Added_Attraction_In_Nassau36339.shtml http://www.bahamashistoricalsociety.com/museum.shtml http://museums-ioj.org.jm/ http://natgalja.org.jm/ http://haitist.com/explore-haitian-art-and-history-at-the-national-museum/ http://www.world66.com/centralamericathecaribbean/thecaribbean/haiti/portauprince/si ghts/thenationalmuseum http://www.arqueotur.org/yacimientos/museo-de-nuestra-raiz-africana-casa-de-loscontrafuertes.html https://centroleon.org.do/cl/ http://www.nichbelize.org/mob/the-museum-of-belize.html http://munae.gob.gt/ http://www.cultura.gob.sv/muna/ https://vianica.com/attraction/458/judith-kain-house-museum http://www.museocostarica.go.cr/es_cr/aportes/la-herencia-afro.html?Itemid=120 http://www.panama-museums.com/en/afro_antillano.aspx 
http://www.museonacional.gov.co/Paginas/default.aspx

https://www.museodeantioquia.co/news/afro-decedents-in-colombian-art/

http://www.alemmar.org/cgibin/quickregister/scripts/redirect.cgi?redirect=EFyZVZAA pkfmAEGgAe

http://www.eluniversal.com/caracas/130401/museo-afroamericano-sera-el-mayor-de-

toda-latinoamerica

http://www.surinaamsmuseum.net/

http://amazonian-museum-network.org/en/amazonian-museums/musee-des-culturesguyanaises

http://amazonian-museum-network.org/en/amazonian-museums/musee-departementalalexandre-franconie

http://www.casadelacultura.gob.ec/?ar_id=3\&mu_id=16\&palabrasclaves=Museo\%20de \%20sitio

http://www.limaeasy.com/culture-guide/museums-lima/national-afroperuvian-museum http://www.museu-goeldi.br/portal/

http://www.museuafro.ufma.br/colecoes.php?tipo_col=5\&acervo=1\#

http://www.guiace.com.br/termo/museu-arthur-ramos

http://mcc.ufrn.br/

http://fundacaogilbertofreyre.blogspot.com.br/

http://www.museudoestadope.com.br/colecao/Cole\%C3\%A7\%C3\%A3o-Afro-do-Xang \%C3\%B4-em-Pernambuco

http://museudaabolicao.museus.gov.br/

http://www.fundaj.gov.br/index.php?option=com_content\&id=250\&Itemid=238

http://www.ufal.edu.br/extensao/equipamentos-culturais/museus/museu-theo-brandao

http://www.cultura.al.gov.br/politicas-e-acoes/patrimonio-cultural/patrimonio-

historico/bens-moveis/colecao-perseveranca

http://www.portalcultura.se.gov.br/homepages/portalcultura.nsf/afbba48c0a268a2b0325

685e0067c3fe/fda6d9dd75d489fa03257068003c6e89!OpenDocument

http://www.mafro.ceao.ufba.br/index.php

http://www.ighb.org.br/\#!museu/c14bv

http://www.institutofeminino.org.br/home/index.php

http://manutencao.ufba.br/

http://www.museucostapinto.com.br/expo_eventos2.asp

http://www.museunacional.ufrj.br/index.html

http://www.cnfcp.gov.br/interna.php?ID_Secao=2

http://mnba.gov.br/

http://www.nptbr.mae.usp.br/

http://www.pinacoteca.org.br/pinacoteca-pt/default.aspx

http://masp.art.br/masp2010/

http://www.museuafrobrasil.org.br/home

http://www.mnba.cl/617/w3-channel.html

http://maam-uruguay.blogspot.com.br/

http://www.doyo.com.ar/museo.htm

Oceania 
http://en.chnmuseum.cn/

http://www.minpaku.ac.jp/english/

http://www.imj.org.il/

http://www.nationalgallery.org/

Europa

http://www.varldskulturmuseerna.se/en/etnografiskamuseet/

http://www.kansallismuseo.fi/en/museum-of-cultures/collections

http://www.kunstkamera.ru/en/collections/collections_on_the_culture_and_life_of_the_ peoples_of_the_world/africa/

http://www.arts-museum.ru/index.php

http://www.erm.ee/en

http://www.varldskulturmuseerna.se/en/etnografiskamuseet/

http://natmus.dk/historisk-viden/verden/afrika/

http://www.museum.ie/

https://www.britishmuseum.org/about_us/departments/africa,_oceania,_americas/histor y_of the collection.aspx

http://www.nhm.ac.uk/

https://www.royalacademy.org.uk/

http://www.liverpoolmuseums.org.uk/mol/

http://www.liverpoolmuseums.org.uk/maritime/

http://www.rmg.co.uk/national-maritime-museum

http://www.prm.ox.ac.uk/

https://www.uea.ac.uk/

http://www.britishmuseum.org/

https://www.wereldmuseum.nl/en/home.html

https://afrikamuseum.nl/

https://tropenmuseum.nl/

https://volkenkunde.nl/nl

http://www.dagjeweg.nl/tip/2227/Afrikacentrum---reis-door-West-Afrika

http://denhaag.com/en/location/7215/gemeentemuseum-den-haag

http://krollermuller.nl/bezoek

http://www.evug.be/

http://www.museel.be/fr

http://www.museedumasque.be/

http://www.routard.com/mag_evenement/395/le_mas_nouveau_musee_d_anvers.htm https://www.uitinvlaanderen.be/agenda/a/etnografisch-museum/31E34EFA-C21F6F48-

EDB166018AD63779

http://www.wittert.ulg.ac.be/

http://www.africamuseum.be/home

http://www.rem-mannheim.de/en/museums/world-cultures-museum/welcome/

http://www.lindenmuseum.de/ 
http://www.ulm.de/ulm_museum/museum/history.110883.3080,110499,110873,110883 $\underline{\text { htm }}$

http://www.weltkulturenmuseum.de/en

http://landesmuseum-koblenz.de/

http://www.museenkoeln.de/rautenstrauch-joest-museum/

http://www.iwalewahaus.uni-bayreuth.de/

http://www.kunst-und-kultur.de/index.php?Action=showMuseum\&mId=476

https://www.krefeld.de/de/textilmuseum/41-deutsches-textilmuseum/

http://www.forum-der-voelker.de/

http://www.braunschweig.de/kultur/museen/staedtisches_museum/index.html

http://www.uebersee-museum.de/ueber-uns/uebersee-museum/

http://www.skd.museum/de/museen-institutionen/japanisches-palais/museum-fuer-

voelkerkunde-dresden/index.html?tx_eventcal_pi1\%5Bdiff\%5D=2

http://www.mvl-grassimuseum.de/sammlungen/

http://www.inyourpocket.com/munich/Staatliches-Museum-fur-Volkerkunde-Munchen-

Ethnology-Museum-_22761v

http://www.voelkerkundemuseum.com/900-0-Africas-Top-Models.html

http://www.zkfl.de/voelkerkundesammlung.html

http://www.landesmuseum-hannover.niedersachsen.de/portal/live.php?navigation

id=24293\&article_id=83589\&_psmand=183

http://www.smb.museum/museen-und-einrichtungen/ethnologisches-museum/

home.html

http://www.naturhistorischesmuseumnuernberg.de/main.php?page=home_starte.php\&s prache $=$ en

http://www.braunschweig.de/kultur/museen/staedtisches_museum/ausstellungen.html

http://www.uebersee-museum.de/en/

http://www.museum-fuenf-kontinente.de/ausstellungen/africa-english/

http://www.ethnomuseum.pl

http://www.nm.cz/

http://www.quaibranly.fr/

http://musees-nationaux-malmaison.fr/musees-napoleonien-africain/

http://vieille-charite-marseille.com/index/le-musee-d-arts-africains-oceaniens-

amerindiens-maaoa

http://musee-angouleme.fr/musee-collections/les-collections/arts-extra-europeens/la-

collection/

https://meb.u-bordeaux.fr/Le-musee

http://mba.caen.fr/

http://fr.viamichelin.ch/web/Site-Touristique/La_Rochelle-17000-

Museum_d_Histoire_Naturelle-a5912k9j

http://www.musee-africain-lyon.org/

http://www.icra.asso.fr/mhnl/gintro.htm

http://www.mam.paris.fr/

https://www.centrepompidou.fr/fr/

http://www.museepicassoparis.fr/en/

http://www.dapper.fr/ 
http://www.museedelhomme.fr/en

http://www.grandpalais.fr/fr

https://www.monnaiedeparis.fr/

http://www.louvre.fr/

http://www.histoire-immigration.fr/

http://www.perigueux-maap.fr/

http://www.allier-auvergne-tourisme.com/culture-patrimoine/musees-spectacles/museedes-arts-d-afrique-et-d-asie-127-1.html

http://www.agglo-niort.fr/-musee-bernard-d-agesci

http://vieille-charite-marseille.com/

http://www.musees-franchecomte.com/index.php?p=296

http://culture.marseille.fr/les-musees-de-marseille/musee-d-arts-africains-oceaniens-

amerindiens-maaoa

http://www.barbier-mueller.ch/?lang=fr

http://institutions.ville-geneve.ch/fr/mah/

http://www.ville-ge.ch/meg/index.php

http://www.men.ch/fr/accueil/

http://www.bhm.ch/en/

http://www.mkb.ch/de/programm.html

http://www.fondationbeyeler.ch/en/Home

http://www.musethno.uzh.ch/en.html

http://www.rietberg.ch/en-gb/home.aspx

http://www.st.gallen.ch/historischesmuseum/

http://www.baden.at/de/unsere-stadt/kultur/rollettmuseum-stadtarchiv/rollettmuseum

/das-rollettmuseum.html

http://www.bergbaumuseum-klagenfurt.at/

http://www.voelkerkundemuseum.com/41-1-Mission-Statement.html

http://www.neprajz.hu/

http://www.infozagreb.hr/?id=94\&l=e\&solo=263

http://www.museoscienzebergamo.it/web/

http://www.msn.unifi.it/collezioni/antropologia-e-etnologia-2-2/

http://sistemamuseo.it/ita/2/musei/30/montone-umbria-museo-etnografico-il-tamburo-

parlante/

http://www.pigorini.beniculturali.it/

http://www.museivaticani.va/2_IT/pages/MV_Visite.html

http://www.060608.it/it/cultura-e-svago/beni-culturali/musei/museo-africano.html

http://www.santafara.org/index.php?option=com_content\&view=article\&id=49

http://www.artesafricanae.org/ita/i-links.htm

http://www.spain.info/pt_BR/que-quieres/arte/museos/madrid/museo_africano_

_mundo_negro.html

http://mnantropologia.mcu.es/

http://www.museusdebanyoles.cat/Default.aspx?alias=www.museusdebanyoles.cat/dard er

http://ajuntament.barcelona.cat/museuetnologic/en 
https://mnetnologia.wordpress.com/

http://www.socgeografialisboa.pt/museu-categorias/africa-2

http://www.museudaciencia.org/

http://www.cm-figfoz.pt/index.php/cultura/2014-03-20-16-23-51/museus

https://sigarra.up.pt/up/pt/web_base.gera_pagina?p_pagina=edif\%C3\%ADcio\%20da

\%20reitoria\%20-\%20museu\%20de\%20hist\%C3\%B3ria\%20natural\%20da\%20u.porto http://www.cm-gaia.pt/gaia/portal/user/anon/page/_GA_E300.psml?categoryOID= 6183808080FE82GC\&contentid=ED83801A80CO\&nl=pt.

Oceania

http://nga.gov.au/

https://www.tepapa.govt.nz/ 